\title{
Considering Peak Rectifier Behaviour for Voltage Distortion Calculations in Power Distribution Systems
}

\author{
Jozef A.L. Ghijselen, member, IEEE, Wouter R.A. Ryckaert, member, IEEE, \\ David P.I. Zenner, and Jan A.A. Melkebeek, senior member, IEEE \\ Ghent University, Department of Electrical Energy, Systems and Automation (EESA), \\ Electrical Energy Laboratory (EELAB) \\ Sint-Pietersnieuwstraat 41, B-9000 Gent, Belgium \\ tel.: (+32).9.264.34.18 fax: (+32).9.264.35.82 e-mail: jozef.ghijselen@rug.ac.be
}

\begin{abstract}
In many cases, power system harmonic studies are performed using current source models for the considered non-linear loads. Peak rectifier loads are known to be highly sensitive to the parameters of the power system impedance and the voltage distortion at the rectifier terminals. The line current spectrum of such rectifiers may be corrected to account for the influence of these parameters. In this paper it is shown that the resulting current source model does not yield sufficiently accurate results when the power system is subjected to resonances.

Keywords-Power Quality, Distribution Systems, Harmonics
\end{abstract}

\section{INTRODUCTION}

The ongoing proliferation of harmonic polluting loads in electric power distribution systems raises increasing concerns. These loads cause harmonic voltage distortion that may propagate throughout the distribution system and into the transmission system. It is well known that voltage waveform distortion may adversely affect the equipment connected to the power system [1].

In particular, harmonic resonance phenomena in power systems are known to cause severe voltage distortion, and even failure of power system components. Harmonic resonance originates from the presence of capacitance in the power system (e.g. from conductor capacitance or power factor correcting capacitors), resonating with the inductive part of the power system impedance (from the transformer short-circuit and conductor series impedances). Non-linear loads are expected to cause a large voltage distortion when their current contains harmonic components with frequencies around the series or parallel resonant frequencies. Their presence requires harmonic mitigation measures [2], [3] to avoid problems.

Power system harmonic studies aim, among other goals, at predicting the amount of harmonic voltages at the different circuit nodes when non-linear loads are present. Unfortunately, power quality studies taking into account the influence of many non-linear loads in LV or MV power systems are quite difficult to undertake. Many non linear-loads, e.g. peak rectifiers, are very sensitive to variations of the power system parameters, such as the power system voltage waveform and power system impedance [4],[5]. The latter may cause harmonic cancelling, which may also originate from phase diversity between the line currents of individual loads [6]. To include the influence of the power system parameters (including harmonic cancelling) ac- curately, the non-linear load current is best predicted by calculations in the time domain, either by time-step simulation (e.g. PSPICE, EMTP) or using analytical models [7]. When the number of loads to be modelled in the power system is large, timestep simulations quickly become unpractical; to ensure numerical accuracy and stability, small time step values are required, rendering the simulation time of large systems extremely long. Moreover, the interaction between the non-linear devices in a power system often introduces convergence problems which are very difficult to solve.

Mixed-mode simulation techniques like iterative harmonic analysis (IHA) model the power system network in the frequency domain and the non-linear loads in the time domain [8]. Because the mutual influence between the non-linear devices is applied in an iterative way, this approach may seem attractive to reduce the calculation time and the risk of convergence problems. Unfortunately, when applied to loads which are highly sensitive to power system parameter variations (such as peak rectifiers), comparable simulation time and convergence problems as with time-step simulations arise.

Consequently, many large-scale power system harmonic studies still resort to modelling the non-linear loads as current sources [9]. Some authors try to account for the effects of harmonic cancelling and apply corrections to the rectifier line current waveform [10] or use measured current waveforms applying an appropriate power system impedance [11].

This paper will show that reasonable harmonic cancelling factors are not sufficient to accurately predict the voltage distortion when non-linear loads are modelled as current sources. Especially when power system resonances occur and highly sensitive non-linear loads (such as peak rectifiers) are present, the expected harmonic voltage levels may be overestimated, and the required power system conditions to provoke resonances are inaccurately predicted.

\section{MODELling APPROACH}

\section{A. Modelling the power system}

For the purpose of this paper, the power system is modelled as a lumped impedance network, which is suitable for representing MV and LV distribution systems. The internal impedance of 


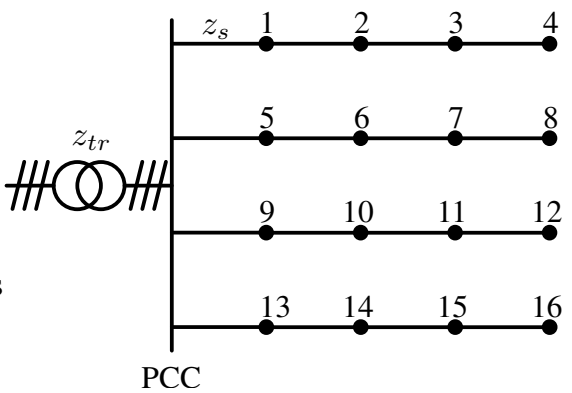

Fig. 1. Radial distribution system under study

the power system voltage source is dominated by the impedance $z_{t r}$ of the transformer feeding the network. The lines interconnecting the network nodes are modelled by resistive/inductive impedances $z_{s}$. The presence of resonance conditions is modelled using capacitor banks compensating linear inductive loads. Cable capacitance is included in the capacitance of capacitor banks.

A three-phase transformer supplying a number of (three-phase) radial distribution feeders with several nodes each is considered (Fig. 1). The transformer impedance $z_{t r}$ and line impedance $z_{s}$ are chosen as follows:

$z_{t r}=0.1 e^{j \frac{4 \pi}{9}} \mathrm{pu}, z_{s}=0.01 e^{j \frac{\pi}{6}} \mathrm{pu}$

The large short-circuit impedance $\left|z_{t r}\right|$ is a typical value for HV/MV transformers to limit the short-circuit power. Because of their large size, the short-circuit impedance is very inductive (typ. $\theta_{t r}=80$ degrees) to limit the full-load losses

The conductor parameters $z_{s}$ vary widely among different distribution systems. The major difference between conductor types is visible in the phase angle $\theta_{s}$ of the conductor impedance, ranging from 30 degrees for cables to 55 degrees for typical overhead lines. Simulations can be performed with and without neutral conductor in the distribution network. The removal of the neutral conductor is accomplished by modelling the connection between the network node and the loads connected to it by an (ideal) $\Delta / Y$ transformer.

\section{B. Modelling the linear loads}

In literature, many linear load models are available, the choice of which is very important for the correct prediction of the frequency and quality factor of resonance conditions [12]. In this paper, linear loads are represented by series RL-circuits, shunted by a capacitance to obtain unity power factor at the fundamental frequency (Fig. 2). Although such a simple representation may not be accurate to model the damping of actual resonance conditions, it is sufficient to show the differences between different modelling approaches for non-linear loads. Different capacitance values (and, hence, different resonance conditions) can be modelled by varying the fundamental power factor $\cos \phi$ of the RL-circuit. A network without resonance (i.e. $\cos \phi=1$ ) is obtained for $x_{l}=0$ and $x_{c}=\infty$.

The load admittance $y_{l}(h)$ as a function of the harmonic order $h$ is then expressed as:

$y_{l}(h)=\frac{1}{r_{l}+j h x_{l}}+j h y_{c} \mathrm{pu}$

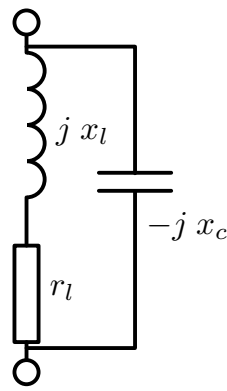

Fig. 2. Linear RL-load model, with power factor correcting capacitor

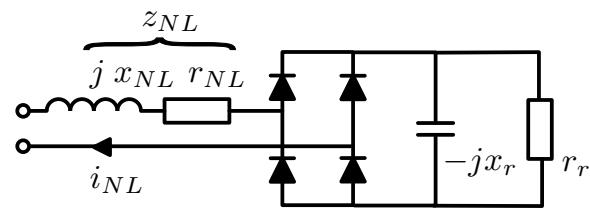

Fig. 3. Rectifier model

where $y_{c}=1 / x_{c}$. Using the pu active power consumption $p_{L}$ of the linear load and the fundamental power factor $\cos \phi$ of the RL-circuit as parameters, it is easily verified that:

$r=\frac{\cos ^{2} \phi}{p_{L}}, x_{l}=r_{l} \tan \phi, y_{c}=p_{L} \tan \phi$

The total linear load $p_{L}$ is lumped into and equally divided among the network nodes, and is symmetrically connected between the phases and the neutral conductor.

\section{Modelling the non-linear loads}

Among the different single-phase loads connected to the distribution system, three non-linear load groups are significantly important [5]. The first group contains the compact fluorescent lamps (CFLs) that employ magnetic ballasts. The second load group utilises phase control of thyristors to control both the input voltage and power to electrical devices, e.g. light dimmers, heating loads and controls for single-phase induction motors. The third load group present in the distribution systems, contains the loads that employ the capacitor filtered diode bridge rectifier (=peak rectifier) as their power supply. The input current waveform is discontinuous since the current is drawn only when the capacitor is charging. This current is rich in harmonics and the total harmonic distortion (THDI) is normally in the range of $100 \%$. Peak rectifiers are widely applied for power levels ranging from a few Watts to several hundred Kilowatts. Examples of such loads include TV sets, personal computers, battery chargers, electronic ballasts for gas discharge lamps and small adjustable speed drives. In this paper, special interest is dedicated to the third load group because of their high line current distortion and their ongoing proliferation in domestic, office and industrial power systems.

These loads can be modelled as single-phase peak rectifiers, as shown in Fig. 3. The rectifier filter impedance $z_{N L}$ may serve to model both the actual filter impedance and/or the local power system impedance caused by the MV/LV transformer and the LV cables. Including two cases for the filter impedance $z_{N L}$, 
the parameters are chosen as follows:

$$
\begin{aligned}
z_{N L}=r_{N L}+j x_{N L} & =0.01 e^{j \frac{\pi}{4}} \mathrm{pu}(\text { actual filter impedance }) \\
& =0.05 e^{j \frac{\pi}{4}} \mathrm{pu}(\text { actual filter impedance }
\end{aligned}
$$
and MV/LV transformer)

$r_{r}=1.5 p_{N L}$

$x_{r}=0.06 \mathrm{pu}$

where the pu values are referred to the nominal load $p_{N L}$ of the rectifier.

The difference between (4) and (5) is caused by the impedance of the MV/LV transformers. The MV/LV transformers usually exhibit a smaller short-circuit impedance (typ. $\left.\left|z_{t r}\right|=0.04 \mathrm{pu}\right)$ than HV/MV transformers, and may be moderately to highly inductive (typ. $\theta_{z_{t r}}=45 \ldots 80$ degrees), depending on the rated apparent power (typ. 10... $1600 \mathrm{kVA}$ ).

The choice of the load resistor $r_{r}$ is not the exact value as required to obtain $p_{N L}$; however, this eliminates the need for power flow calculations and iterations on $r_{r}$, and provides sufficient accuracy for the purpose of this paper [13].

The total rectifier load $p_{N L}$ is equally divided among the network nodes, and in every network node a symmetrical threephase rectifier load is considered, by connecting three singlephase rectifiers between the phases and the neutral conductor.

The rectifier load behaves more like a current source for lower values of $p_{N L}$ and for higher values of $\left|z_{N L}\right|$. Indeed, if the $\mathrm{MV} / \mathrm{LV}$ transformers are substituted by ideal 1/1 transformers and if the conversion of the MV impedances to the LV voltage is taken into account, it is easily verified that:

$Z_{t r}=z_{t r} \cdot Z_{r e f}$

$Z_{N L}=z_{N L} \cdot \frac{V_{L S}^{2}}{S_{N L}}=z_{N L} \cdot \frac{V_{L S}^{2}}{\frac{S_{M S} \cdot p_{N L}}{3 \cdot N}}=\frac{z_{N L} \cdot Z_{r e f} \cdot N}{p_{N L}}$

with

$$
\left\{\begin{array}{l}
V_{r e f}=V_{L S}=\text { phase voltage } \\
S_{r e f}=S_{M S}=3 \text {-ph apparent power of the HV/MV transformer } \\
Z_{r e f}=\frac{3 \cdot V_{L S}^{2}}{S_{M S}} \\
Z_{t r}(h)=\left|Z_{t r}\right| \cdot\left(\cos \phi_{t r}+j \cdot h \cdot \sin \phi_{t r}\right) \\
Z_{N L}(h)=\left|Z_{N L}\right| \cdot\left(\cos \phi_{N L}+j \cdot h \cdot \sin \phi_{N L}\right) \\
N=\text { number of network nodes }
\end{array}\right.
$$

For $h>2$, the impedances are highly inductive and the ratio

$\frac{Z_{N L}}{Z_{t r}} \approx \frac{\left|z_{N L}\right| \cdot N \cdot \sin \left(\phi_{N L}\right)}{\left|z_{t r}\right| \cdot p_{N L} \cdot \sin \left(\phi_{t r}\right)}$

is a measure of the rectifier filter harmonic impedance $Z_{N L}$ as compared to the transformer impedance at the same harmonic order. The greater the value of this ratio, the less sensitive the rectifier becomes for distorted voltages, i.e. current source behaviour.

The ratio $\frac{Z_{N L}}{Z_{t r}}$ for $p_{N L}=0.1$ and $p_{N L}=0.5$ are mentioned in Table I for the considered parameters of $\left|z_{N L}\right|$ and $\left|z_{t r}\right|$. Notice that the values are valid for all harmonics. It follows from $\mathrm{Ta}-$ ble I that the rectifier loads behaves more like a current source for lower values of $p_{N L}$ and for higher values of $\left|z_{N L}\right|$.
TABLE I

THE RATIO $\frac{Z_{N L}}{Z_{t r}}$ FOR $p_{N L}=0.1$ AND $p_{N L}=0.5$ FOR THE CONSIDERED PARAMETERS OF $\left|z_{N L}\right|$ AND $\left|z_{t r}\right|$.

\begin{tabular}{|c||c|c|}
\hline \multicolumn{1}{|c||}{$\left|z_{N L}\right|$} & \multicolumn{2}{c|}{$p_{N L}(\mathrm{pu})$} \\
$(\mathrm{pu})$ & 0.1 & 0.5 \\
\hline 0.01 & 11.5 & 2.3 \\
0.05 & 57.4 & 11.5 \\
\hline
\end{tabular}

\section{Calculation of voltage THD at the PCC}

In the following, the resulting voltage distortion at the point of common coupling (PCC) of the network in Fig. 1 is predicted using different models for the non-linear loads:

1. the non-linear load is modelled in the time domain, using a non-linear electrical circuit simulator;

2. the non-linear load is modelled as a current source, the spectrum of which is obtained from a time-step simulation in which the rectifier (including its filter impedance) is supplied with a purely sinusoidal voltage;

3. the non-linear load is modelled as a current source, the spectrum of which equals the line current spectrum of the rectifier in a node most remote from the PCC (node 4, 8, 12 or 16 of the network). The spectrum is calculated from a time-step simulation of the power network with the linear and non-linear loads equally divided among the network nodes. Also, the $\cos \phi$ of the linear loads is set to unity, thus no resonances are present in the power system. This approach provides a current waveform reasonably accounting for harmonic attenuation due to the power system impedance.

All calculations include a sweep of the $\cos \phi$ of the RL-circuits between 0.8 and 1 , as to predict the influence of different resonance conditions on the voltage distortion.

\section{Calculations}

\section{A. Time-step simulations}

The THD of the voltage at the PCC is now calculated using time-step simulations, for different load parameters. Simulations are performed for a non-linear load $p_{N L}$ of 0.1 and 0.5 pu and filter impedances $\left|z_{N L}\right|$ of 0.01 and $0.05 \mathrm{pu}$. In any case, the total active power load of the network equals $1 \mathrm{pu}$, therefore, the linear load $p_{L}$ equals $1-p_{N L}$. The $\cos \phi$ of the RL-circuits is swept from 0.8 to 1 . For every local maximum of the voltage THD at the PCC, the voltage harmonics with correlating maxima are given. This is illustrated in Figures. 4 and 5. In Fig. 4, the $T H D\left(v_{P C C}\right)$ is given for $p_{N L}=0.1 \mathrm{pu}$ and $\left|z_{N L}\right|=0.05$ $\mathrm{pu}$, neutral wire present. For this case, $T H D\left(v_{P C C}\right)$ shows maxima at $\cos \phi$ equalling $0.98,0.95$ and 0.86 , clearly correlating with the maxima of the 9 th, 7 th resp. 5th harmonic of $v_{P C C}$. From the four considered rectifier types, this configuration approaches mostly the current source behaviour (See section II-C). The results for other cases as well as for $\cos \phi=1$ are summarised in Table II. From this table it can be noticed that a maximum of $T H D\left(v_{P C C}\right)$ may correlate with maxima of more than one harmonic. This is due to the non-linear behaviour of the rectifier, and is more pronounced when the rectifier load behaves less like a current source, i.e. for higher values of $p_{N L}$ and lower values of $\left|z_{N L}\right|$. This is illustrated in Fig. 5, where $T H D\left(v_{P C C}\right)$ 


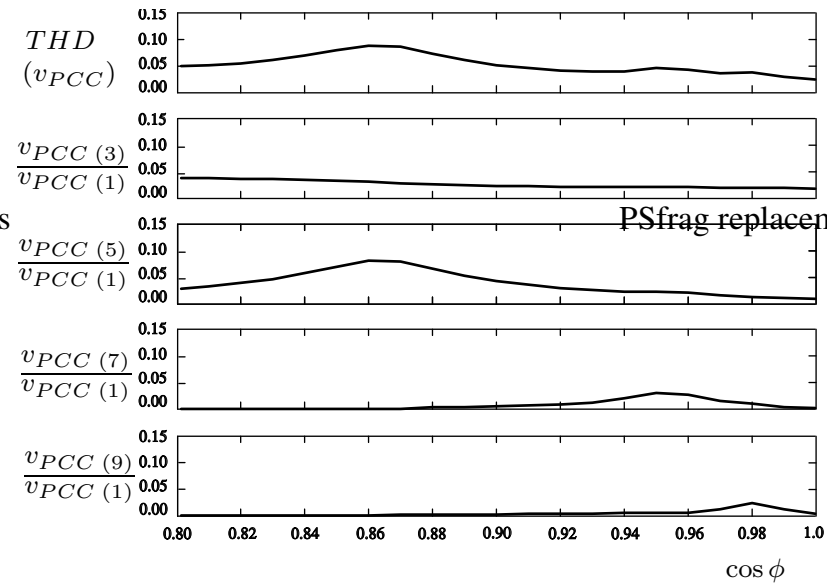

Fig. 4. Time-step calculations of the voltage THD at the PCC, and its dominant harmonics for $p_{N L}=0.1 \mathrm{pu}$ and $\left|z_{N L}\right|=0.05 \mathrm{pu}$, neutral wire present

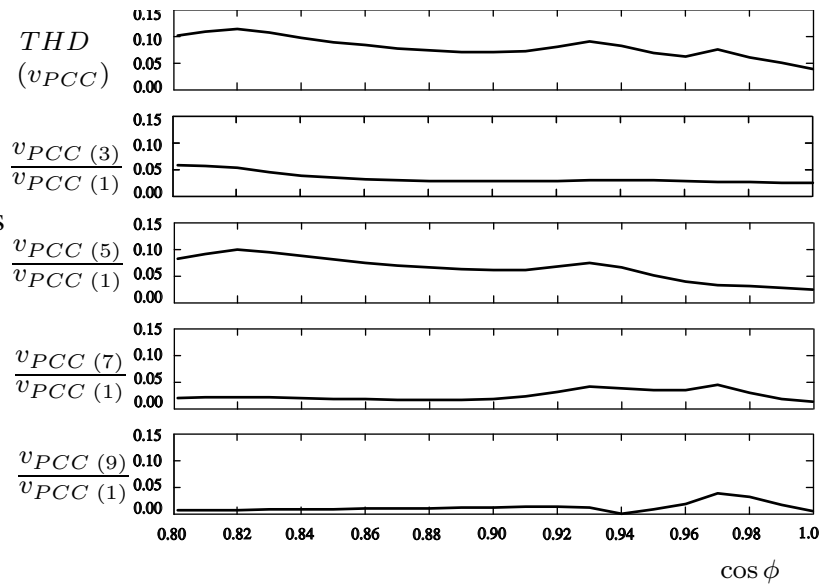

Fig. 5. Time-step calculations of the voltage THD at the PCC, and its dominant harmonics for $p_{N L}=0.1 \mathrm{pu}$ and $\left|z_{N L}\right|=0.01 \mathrm{pu}$, neutral wire present

TABLE II

VolTAGE THD [\%] AT THE PCC FOR TIME-STEP SIMULATIONS

\begin{tabular}{|c|c|c|c|c|c|c|c|c|}
\hline \multirow{3}{*}{$\begin{array}{c}z_{N L}\left(\text { pu re. } p_{N L}\right) \\
p_{N L}(\mathrm{pu}) \\
\text { neutral }\end{array}$} & \multicolumn{4}{|c|}{$0.01 e^{j \pi / 4}$} & \multicolumn{4}{|c|}{$0.05 e^{j \pi / 4}$} \\
\hline & \multicolumn{2}{|c|}{0.1} & \multicolumn{2}{|c|}{0.5} & \multicolumn{2}{|c|}{0.1} & \multicolumn{2}{|l|}{0.5} \\
\hline & Yes & No & Yes & No & Yes & No & Yes & No \\
\hline $\cos \phi$ & & & & & & & & \\
\hline 1.00 & 3.93 & 3.05 & 12.36 & 8.97 & 2.37 & 1.38 & 9.57 & 5.81 \\
\hline $\mathrm{h}$ & - & - & - & - & - & - & - & - \\
\hline 0.98 & - & 5.31 & - & - & 3.69 & - & - & 13.08 \\
\hline $\mathrm{h}$ & - & 11,13 & - & - & 9 & - & - & 11,13 \\
\hline 0.97 & 7.50 & - & - & 15.18 & - & - & - & - \\
\hline $\mathrm{h}$ & $7,9,11,13$ & - & - & $7,11,13$ & - & - & - & - \\
\hline 0.96 & - & - & - & - & - & - & 17.69 & - \\
\hline $\mathrm{h}$ & - & - & - & - & - & - & $7,9,11,13$ & - \\
\hline 0.95 & - & - & 22.45 & - & 4.66 & 3.86 & - & 13.22 \\
\hline $\mathrm{h}$ & - & - & $7,9,13$ & - & 7 & 7 & - & 11,13 \\
\hline 0.94 & - & 8.20 & - & - & - & - & - & - \\
\hline $\mathrm{h}$ & - & 5,7 & - & - & - & - & - & - \\
\hline 0.93 & 9.14 & - & - & 19.34 & - & - & - & - \\
\hline $\mathrm{h}$ & 5,7 & - & - & 5,7 & - & - & - & - \\
\hline 0.91 & - & - & - & - & - & - & 21.59 & - \\
\hline $\mathrm{h}$ & - & - & - & - & - & - & $5,7,9,13$ & - \\
\hline 0.90 & - & - & 21.81 & - & - & - & - & - \\
\hline $\mathrm{h}$ & - & - & $5,7,9,13$ & - & - & - & - & - \\
\hline 0.86 & - & - & - & - & 8.80 & 9.06 & - & - \\
\hline $\mathrm{h}$ & - & - & - & - & 5 & 5 & - & - \\
\hline 0.84 & - & - & 22.65 & - & - & - & - & 26.59 \\
\hline $\mathrm{h}$ & - & - & $5,7,13$ & - & - & - & - & 5,7 \\
\hline 0.82 & 11.50 & 10.00 & - & - & - & - & - & - \\
\hline $\mathrm{h}$ & 5 & 5 & - & - & - & - & - & - \\
\hline 0.81 & - & - & - & - & - & - & 24.85 & - \\
\hline $\mathrm{h}$ & - & - & - & - & - & - & 5,7 & - \\
\hline
\end{tabular}

is given for $p_{N L}=0.1 \mathrm{pu}$ and $\left|z_{N L}\right|=0.01 \mathrm{pu}$, neutral wire present. The maximum of $T H D\left(v_{P C C}\right)$ at $\cos \phi$ equalling 0.93 , correlates with a local maximum of the 5 th and the 7 th harmonic voltage component.

\section{B. General considerations on current source modelling}

When the non-linear loads are modelled as current sources, the network becomes linear and distinct resonance frequencies can be correlated with distinct $\cos \phi$ values. The resonance frequency is mainly determined by the parallel resonance of the (inductive) transformer impedance $z_{t r}$ and the reactance $x_{c}$ of the shunt capacitors, compensating the linear loads. From equation (3) it follows that the reactance $x_{c}$ is dependent on both $p_{L}$ and $\cos \phi$. The resulting resonance frequencies and the corresponding $\cos \phi$ values are summarised in Table III. Comparison with Table II readily shows that the $\cos \phi$ values for resonance at a given harmonic do not correlate between time-step simulations and current source calculations. This is due to both the effect of the rectifier filter impedance $z_{N L}$, influencing the power system impedance, and the non-linear behaviour of the rectifier which can only be approximated by a current source model. Also, from Table III it follows that the maxima for the THD 
TABLE III

REQUIRED $\cos \phi$ TO OBTAIN RESONANCE CONDITION AT A GIVEN HARMONIC $h$ WHEN CURRENT SOURCE MODELLING IS APPLIED

\begin{tabular}{|c||c|c|}
\hline \multicolumn{1}{|c||}{} & \multicolumn{2}{c|}{$p_{N L}(\mathrm{pu})$} \\
$\mathrm{h}$ & 0.1 & 0.5 \\
\hline 5 & 0.884 & 0.754 \\
7 & 0.960 & 0.910 \\
9 & 0.982 & 0.961 \\
11 & 0.990 & 0.980 \\
13 & 0.994 & 0.989 \\
\hline
\end{tabular}

correlate with a single harmonic maximum, contrasting with the multiple harmonic maxima found in Table II for time-step simulations. Concluding, current source modelling cannot provide an accurate prediction of the required network conditions provoking resonances.

\section{Current source modelling: matching with purely sinusoidal voltage supply}

By time-step simulations, the line current waveform of the peak rectifier (including its filter impedance) at purely sinusoidal voltage supply is obtained. This approach does not take into account any effects of diversity, which refers to the partial harmonic cancellation of the currents due to the dispersion in harmonic current phase angles, and of attenuation, which refers to the interaction of the load voltage and current distortion. Therefore, this approach represents the rectifier loads with an unrealistic and high current THD.

The non-linear loads are now replaced by current sources with the same spectrum. The resulting voltage THD at the PCC is shown in Table IV, and in Figures 6 and 7 for the particular cases of $p_{N L}=0.1 \mathrm{pu}$, neutral conductor present. The large amount by which the resulting PCC voltage THD is overestimated is clearly visible, and is caused by the unrealistic current source THD and by neglecting the effects of diversity and attenuation. For the particular case of $p_{N L}=0.1 \mathrm{pu},\left|z_{N L}\right|=0.01 \mathrm{pu}$ and $h=5$, the overestimation of the PCC voltage THD is $238 \%$.

Two important differences are noticed with the time-step simulations of the network:

- Comparing Table II and Table III, the maxima of the voltage THD at the PCC occur at different values of the $\cos \phi$ of the linear loads. Using the current source modelling approach, the impedance matrix of the network shows clear evidence of resonance conditions at the voltage THD maxima, as was explained in the previous paragraph. Analysing the spectrum of the PCC voltage from the time-step simulations, the voltage THD maxima also occur at resonance conditions; however, for a given harmonic, resonance occurs at lower $\cos \phi$ values than predicted by the current source approach. Comparison of Fig. 4 with Fig. 6 and Fig. 5 with Fig. 7 readily leads to this conclusion. This is mainly explained by the fact that the (inductive) rectifier filters reduce the (inductive) power system impedance, therefore requiring larger capacitances (and hence lower $\cos \phi$ values) to fulfill the resonance condition for that harmonic.

The greater the impedance $\left|z_{N L}\right|$ (inductive), the more the rectifier load behaves like a current source (Section II-C), and consequently, the smaller the difference becomes between the current source modelling approach and the time-step simulations.
- The maximum values of the voltage THD at the PCC are much larger for the current source modelling approach. This is explained by the unrealistic THD of the current sources, and the fact that power system voltage distortion at resonance increases severely, affecting the commutation behaviour of the rectifier and, consequently, reduces its line current distortion and causes harmonic cancelling.

\section{Current source modelling: matching with the most remote} node at $\cos \phi=1$

By time-step simulations, the line current waveforms of the peak rectifiers in the different nodes of the network are obtained. The $\cos \phi$ of the linear loads is set to unity. The peak rectifier located at the node which is most remote from the PCC (node 16) shows the lowest line current THD, because the power system impedance in that node is the largest. Therefore, the line current spectrum of this rectifier includes some effects of attenuation and diversity in a reasonable way. When applied to current source modelling, one would expect the current source THD to be slightly underestimated, because the power system impedance in that node is the highest of the network. When the rectifier loads are replaced by a current source with the same waveform, however, the differences with the time-step simulations are of the same nature as explained in the previous paragraph. The results are summarised in Table V. The overestimation of the PCC voltage THD for the particular case of $p_{N L}=0.1 \mathrm{pu},\left|z_{N L}\right|=0.01 \mathrm{pu}$ and $h=5$, is now $177 \%$.

Although the PCC voltage THD is much overestimated in most cases, the difference with time-step simulations is smaller than reported in the previous paragraph. The more the rectifier load behaviour approaches current source behaviour (i.e. for smaller rectifier loads $p_{N L}$ and for higher rectifier filter impedance $\left.\left|z_{N L}\right|\right)$, the smaller the difference with time-step simulation results becomes.

\section{E. Influence of the neutral conductor}

In the previous sections, the results are explained for a power system with a neutral conductor both in the MV and LV distribution system network. The modelling of the neutral conductor is a very simplified representation because the considered neutral conductor has no impedance. Consequently, the zerosequence impedance of the conductors equals the positive- and negative-sequence impedances. This is valid for a three-phase, four-wire arrangement with multi-grounded neutral conductor or for a conductor arrangement where the three-phase conductors are split into three single-phase, two-wire, conductor sets. The removal of the neutral conductor is accomplished by modelling the connection between the network node and the loads connected to it by an (ideal) $\Delta / Y$ transformer. From Tables II, IV and V, it follows that the influence on the voltage THD at the $\mathrm{PCC}$ is relative small. Although there is a strong 3-th harmonic current $( \pm 80 \%)$, it follows from Table II that the influence on the voltage THD at the PCC is relative small because the 3-th harmonic impedance is small, $z_{P C C}(h)=h . z_{P C C}(1)$, in comparison with higher harmonics. The reduction of the 3-th harmonic current seems to be more important to reduce the losses in the neutral conductor than to prevent too high voltage distortion in the power system. 


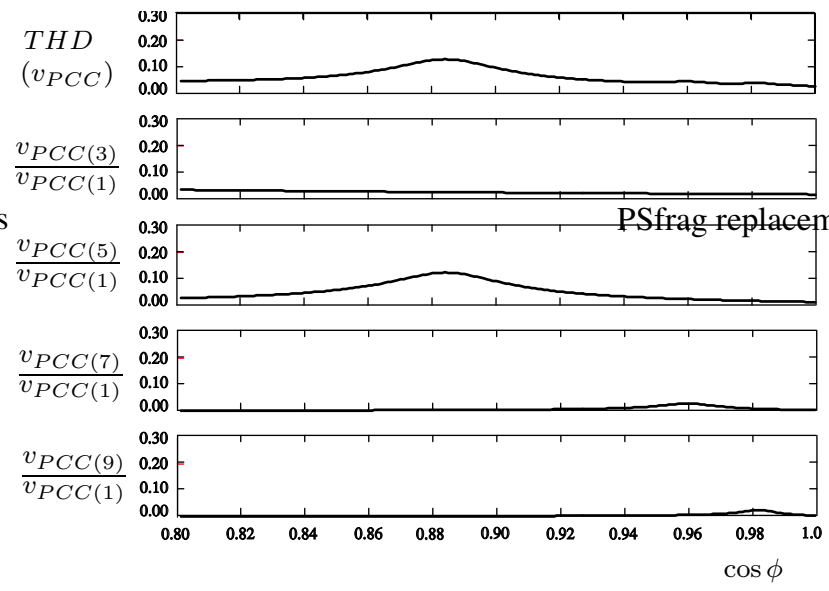

Fig. 6. Current source modelling of the voltage THD at the PCC, and its dominant harmonics for $p_{N L}=0.1 \mathrm{pu}$ and $\left|z_{N L}\right|=0.05 \mathrm{pu}$, neutral wire present

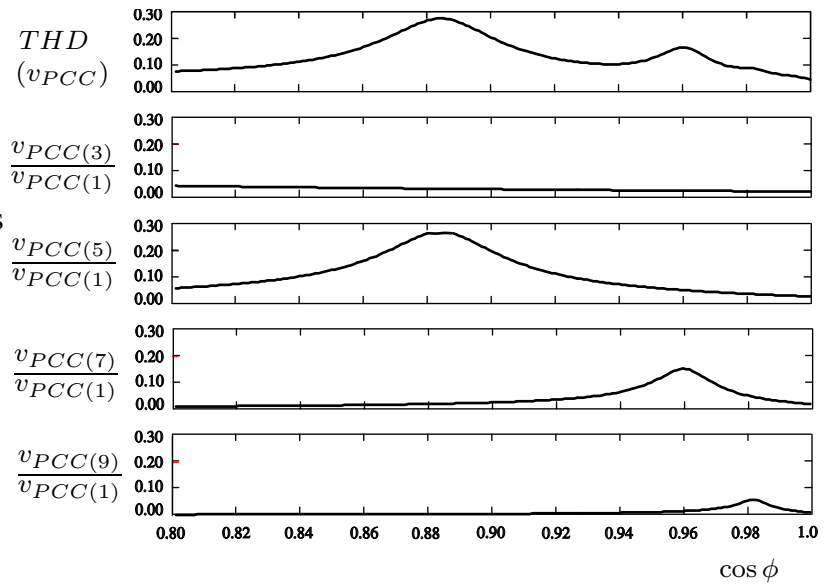

Fig. 7. Current source modelling of the voltage THD at the PCC, and its dominant harmonics for $p_{N L}=0.1 \mathrm{pu}$ and $\left|z_{N L}\right|=0.01 \mathrm{pu}$, neutral wire present

TABLE IV

VOLTAGE THD [\%] AT THE PCC FOR CURRENT SOURCE MODELLING FROM PURELY SINUSOIDAL VOLTAGE SUPPLY (THE REQUIRED COS $\phi$ VALUES TO OBTAIN RESONANCE CONDITION AT A GIVEN HARMONIC $h$ ARE MENTIONED IN TABLE III)

\begin{tabular}{|c|c|c|c|c|c|c|c|c|}
\hline$z_{N L}\left(\right.$ pu re. $\left.p_{N L}\right)$ & \multicolumn{4}{|c|}{$0.01 e^{j \pi / 4}$} & \multicolumn{4}{|c|}{$0.05 e^{j \pi / 4}$} \\
\hline$p_{N L}(\mathrm{pu})$ & \multicolumn{2}{|c|}{0.1} & \multicolumn{2}{|c|}{0.5} & \multicolumn{2}{|c|}{0.1} & \multicolumn{2}{|c|}{0.5} \\
\hline neutral & Yes & No & Yes & No & Yes & No & Yes & No \\
\hline$T H D\left(i_{N L}\right)(\%)$ & \multicolumn{4}{|c|}{$113.79 \%$} & \multicolumn{4}{|c|}{$75.10 \%$} \\
\hline \multicolumn{9}{|l|}{$h$} \\
\hline$(\cos \phi=1)$ & 4.56 & 3.64 & 24.87 & 20.06 & 2.48 & 1.41 & 13.13 & 7.72 \\
\hline 13 & - & - & 42.76 & 38.12 & - & - & 19.69 & 15.70 \\
\hline 11 & - & - & 45.86 & 40.07 & - & 2.01 & 22.14 & 17.73 \\
\hline 9 & 8.94 & - & 87.31 & - & 3.91 & - & 39.29 & - \\
\hline 7 & 16.58 & 16.27 & 188.20 & 187.40 & 4.40 & 3.73 & 38.84 & 36.80 \\
\hline 5 & 27.39 & 27.16 & 241.67 & 240.94 & 12.67 & 12.35 & 110.78 & 109.78 \\
\hline
\end{tabular}

TABLE V

VOLTAGE THD [\%] AT THE PCC FOR CURRENT SOURCE MODELLING FROM THE MOST REMOTE NODE AT COS $\phi=1$ (THE REQUIRED COS $\phi$ VALUES TO OBTAIN RESONANCE CONDITION AT A GIVEN HARMONIC $h$ ARE MENTIONED IN TABLE III)

\begin{tabular}{|c|c|c|c|c|c|c|c|c|}
\hline$z_{N L}\left(\right.$ pu re. $\left.p_{N L}\right)$ & \multicolumn{4}{|c|}{$0.01 e^{j \pi / 4}$} & \multicolumn{4}{|c|}{$0.05 e^{j \pi / 4}$} \\
\hline$p_{N L}(\mathrm{pu})$ & \multicolumn{2}{|c|}{0.1} & \multicolumn{2}{|c|}{0.5} & \multicolumn{2}{|c|}{0.1} & \multicolumn{2}{|c|}{0.5} \\
\hline neutral & Yes & No & Yes & No & Yes & No & Yes & No \\
\hline$T H D\left(i_{N L}\right)(\%)$ & 93.53 & 95.22 & 66.07 & 75.72 & 70.34 & 72.64 & 57.77 & 66.35 \\
\hline \multicolumn{9}{|l|}{$h$} \\
\hline$(\cos \phi=1)$ & 3.37 & 2.55 & 11.28 & 7.62 & 2.28 & 1.28 & 9.50 & 5.46 \\
\hline 13 & - & - & 16.79 & 16.49 & - & - & 13.19 & 10.35 \\
\hline 11 & 4.66 & 4.04 & 20.81 & 23.22 & - & 1.84 & 17.97 & 13.34 \\
\hline 9 & - & - & 30.83 & - & 3.54 & - & 20.63 & - \\
\hline 7 & 8.75 & 9.04 & 43.79 & 35.20 & 4.13 & 3.37 & 41.38 & 34.61 \\
\hline 5 & 20.38 & 20.73 & 77.03 & 105.57 & 10.61 & 11.20 & 49.84 & 73.83 \\
\hline
\end{tabular}

The conclusions of previous sections remain and time simulations are necessary to accurately predict both the resonance conditions and the voltage THD in the PCC and in other network nodes.

\section{CONCLUSION}

In this paper is was shown that current source modelling and reasonable harmonic cancelling factors are not sufficient to accurately predict the voltage distortion when non-linear loads are modelled as current sources. Especially when power system resonances occur and highly sensitive loads (such as peak rectifiers) are present, expected harmonic voltage levels may be overes- timated, and the required power system conditions to provoke resonances are inaccurately predicted.

\section{ACKNOWLEDGEMENTS}

The research of Jozef Ghijselen is partially supported by the Fund for Scientific Research Flanders (FWO-Vlaanderen).

The research of Wouter Ryckaert is supported by the Institute for the Promotion of Innovation by Science and Technology in Flanders (IWT). 


\section{REFERENCES}

[1] IEEE Task Force on the Effect of Harmonics on Equipment, "IEEE Effects of harmonics on equipment," IEEE Transactions on Power Apparatus and Systems, vol. 109, no. 3, pp. 2460-2479, Sept. 1984.

[2] H. Akagi, "New trends in active power line conditioners," IEEE Transactions on Industry Applications, vol. 32, no. 6, pp. 1312-1322, Nov./Dec 1996.

[3] H. Akagi, H. Fujita, and K. Wada, "A shunt active filter based on voltage detection for harmonic termination of a radial power distribution line," IEEE Transactions on Industry Applications, vol. 35, no. 3, pp. 638-645, May/June 1999.

[4] A. Mansoor, W.M. Grady, R.S. Thallam, M.T. Doyle, S.D. Krein, and M.J. Samotyj, "Effect of supply voltage harmonics on the input current of single-phase diode rectifier loads," IEEE Transactions on Power Delivery, vol. 10, no. 3, pp. 1416-1422, July 1995.

[5] El-Saadany E.F. and Salama M.M.A., "Reduction of the net harmonic current produced by single-phase non-linear loads due to attenuation and diversity effects," Electrical Power \& Energy Systems, vol. 20, no. 4, pp. 259-267, May/June 1998.

[6] S. Hansen, P. Nielsen, and F. Blaabjerg, "Harmonic cancellation by mixing nonlinear single-phase and three-phase loads," IEEE Transactions on Industry Applications, vol. 36, no. 1, pp. 152-159, Jan./Feb. 2000.

[7] A. Mansoor, W.M. Grady, A.H. Chowdhury, and M.J. Samotyj, "An investigation of harmonics attenuation and diversity among distributed singlephase power electronic loads," IEEE Transactions on Power Delivery, vol. 10, no. 1, pp. 467-473, Jan. 1995.

[8] R. Carbone, M. Fantauzzi, F. Gagliardi, and A. Testa, "Some considerations on the iterative harmonic analysis convergence," IEEE Transactions on Power Delivery, vol. 8, no. 2, pp. 487-495, Apr. 1993.

[9] D.J. Pileggi, E.M. Gulachensky, C.E. Root, T.J. Gentile, and A.E. Emanuel, "The effect of modern compact fluorescent lights on voltage distortion," IEEE Transactions on Power Delivery, vol. 8, no. 3, pp. 14511457, July 1993.

[10] R. Dwyer, A.K. Khan, R.K. McCluskey, and R. Sung, "Evaluation of harmonic impacts from compact fluorescent lights on distribution systems," IEEE Transactions on Power Systems, vol. 10, no. 4, pp. 1772-1779, Nov. 1995.

[11] R.R. Verderber, O.C. Morse, and W.R. Alling, "Harmonics from compact fluorescent lamps," IEEE Transactions on Industry Applications, vol. 29, no. 3, pp. 670-674, May/June 1993.

[12] A. Capasso, R. Lamedica, R. Manigrasso, G. Sani, G. Superti Furga, and E Tironi, "Reference power network for the harmonic propagation analysis," European Transactions on Electrical Power, vol. 2, no. 3, pp. 167178, May/June 1992.

[13] A. Mansoor, W.M. Grady, P.T. Staats, R.S. Thallam, M.T. Doyle, and M.J. Samotyj, "Predicting the net harmonic currents produced by large numbers of distributed single-phase computer loads," IEEE Transactions on Power Delivery, vol. 10, no. 4, pp. 2001-2006, Oct. 1995

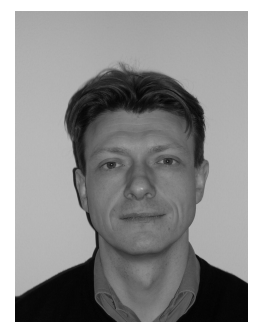

Jozef A.L. Ghijselen (S'89-M'90) received the M.Sc. and $\mathrm{Ph} . \mathrm{D}$. degrees from Ghent University, Belgium, in 1990 and 2001, respectively. Since then, he has been with the Electrical Energy Laboratory (EELAB), Department of Electrical Energy, Systems and Automation (EESA) of Ghent University. His research interests include power electronics and power quality.

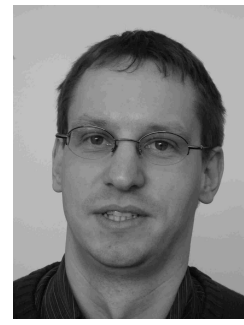

Wouter R.A. Ryckaert (M'02) received the degree of industrial engineer in electrical engineering from Kaho Sint-Lieven, Belgium, in 1998 and the M.Sc. degree in electrical and mechanical engineering from Ghent University, Belgium, in 2001. Since then, he has been with the Electrical Energy Laboratory (EELAB), Department of Electrical Energy, Systems and Automation (EESA) of Ghent University and is currently working towards a $\mathrm{Ph} . \mathrm{D}$. degree. His research interests include power quality and power electronics.

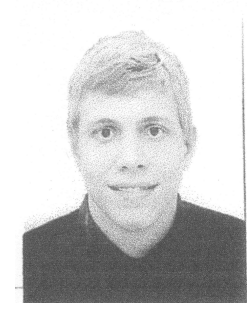

David P.I. Zenner was born in Ghent, Belgium on October 23, 1979. He received the M.Sc. degree in electrical and mechanical engineering from Ghent University, Belgium, in 2001. Since then, he is following a postgraduate course in Electrical Power Engineering at Swiss Federal Institute of Technology, Lausanne (EPFL)

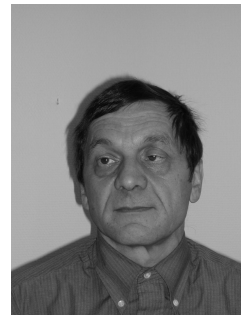

Jan A.A. Melkebeek (M'75-SM'83) received the Dr.Ing. degree in electrical and mechanical engineering, the Ph.D. degree in applied sciences, and the Dr.Habilitus degree in electrical and electronical power technology from Ghent University, Belgium, in 1975, 1980, and 1986, respectively. Since 1987, he has been a Professor in electrical engineering with the Engineering Faculty, Ghent University. His teaching activities and research interests include electrical machines, power electronics, variable frequency drives, and also control systems theory applied to electrical 